SCIENTIFIC REPORT

\title{
Blepharokeratoconjunctivitis in children: diagnosis and treatment
}

\author{
M Viswalingam, S Rauz, N Morlet, J K G Dart
}

Br J Ophthalmol 2005;89:400-403. doi: 10.1136/bjo.2004.052134

\begin{abstract}
Aim: Blepharokeratoconjunctivitis (BKC) is a poorly described entity in children. This study characterises this syndrome in childhood and evaluates epidemiology, clinical grading, and treatment strategies.

Methods: 44 children ( 20 white, 22 Asian, 2 Middle Eastern, median age 5.4 (range 1-14) years) with a diagnosis of BKC were followed for a median of 7 years. Diagnostic criteria included recurrent episodes of chronic red eye, watering, photophobia, blepharitis including recurrent styes or meibomian cysts, and a keratitis. Clinical features were graded as mild, moderate, or severe. The lids and conjunctiva were cultured. The treatment regimen incorporated lid hygiene, topical and/or systemic antibiotics, and topical corticosteroids.

Results: The disease was most severe in the Asian and Middle Eastern children ( $<0.001$ ), who had a statistically higher risk of subepithelial punctate keratitis $(p=0.008)$, corneal vascularisation ( $<0.001)$, and marginal corneal ulcerations $(p=0.003)$, than the white group. 15 children had culture positive lid swabs. Most children had a reduction in symptoms and signs with treatment, and progression of disease after the age of 8 was rare.

Conclusions: BKC in children can be defined as "a syndrome usually associated with anterior or posterior lid margin blepharitis, accompanied by episodes of conjunctivitis, and a keratopathy including punctate erosions, punctate keratitis, phlyctenules, marginal keratitis, and ulceration." BKC is common in children in a tertiary referral corneal and external diseases clinic, with the more severe manifestations in the Asian and Middle Eastern populations. Therapy is effective and loss of sight can be prevented in most cases.
\end{abstract}

$E^{2}$ yelid margin disease with secondary conjunctival and corneal involvement is well characterised in adults and is termed blepharokeratoconjunctivitis (BKC). ${ }^{12}$ Clinical findings typically consist of inflamed eyelids, anterior lid margin telangiectasia, and accumulations of hard, fibrinous, and crusting scales (collarettes) around the base of the cilia. In chronic cases, ulceration, lid notching (tylosis), thinning or loss of lashes (madarosis), or misdirection of lashes (trichiasis) are common. Conjunctival changes in longstanding cases include mild to moderate papillary and follicular hypertrophy of the palpebral conjunctiva. In acute exacerbations, additional inferior corneal involvement (punctate epithelial erosions, marginal infiltrates, sterile marginal abscesses) is also seen. Adult BKC is more responsive to treatment than other forms of chronic blepharoconjunctivitis, the regimen involving lid hygiene together with the use of topical antibiotics. ${ }^{3}$

By contrast, the syndrome of childhood BKC is poorly defined..$^{5}$ In our study, we characterise BKC in children, and evaluate demographics, clinical features, time course, and treatment strategies.

\section{PATIENTS AND METHODS}

Forty four children with a diagnosis of chronic or recurrent BKC were identified from the children presenting to the corneal and external diseases clinic at Moorfields Eye Hospital, London. This hospital is a tertiary referral centre, and has a local catchment area of mixed ethnic origins (white, Asian (Indo-Pakistani), African-Caribbean, Middle Eastern, oriental).

BKC was defined as "a syndrome usually associated with anterior or posterior lid margin blepharitis, accompanied by episodes of conjunctivitis, and a keratopathy including punctate erosions, punctate keratitis, phylctenules, marginal keratitis, and ulceration." The diagnosis of BKC required the following features: recurrent episodes of chronic red eye, watering, photophobia, blepharitis including recurrent styes or meibomian cysts, and a keratitis. ${ }^{12}$ Children with a history of atopy, perennial allergic conjunctivitis, or vernal keratoconjunctivitis were excluded from the study. The children were followed from presentation to discharge, with frequency of follow up dictated by the disease course. Clinical symptoms and signs were graded as mild, moderate, or severe as detailed in table 1 , and figure 1 . Swabs were taken with cotton wool tipped swab sticks; these were dipped in tryptic digest broth for the lid margins and used plain for the lower tarsal conjunctiva. Both swabs were plated onto separate blood agar plates for culture and sensitivity studies.

\section{Treatment}

The children were treated with a regimen similar to that used in adult BKC. Daily lid hygiene was directed at removing debris and crusts along the lid margin, using cotton wool tipped swab sticks with diluted bicarbonate solution, diluted baby shampoo, or warm water. The child's attendant was advised that the lid hygiene routine had to be maintained for an indefinite period.

Patients with mild or moderate disease were prescribed a short course of topical broad spectrum antibiotics, usually chloramphenicol. Drops were instilled four times daily and ointment applied to the lid margins at night for 1 month, after which they were instructed to use only the ointment at night for a further 2-3 months while maintaining a daily routine of lid hygiene. These patients were examined at 1, 2, 3 , and 6 months following presentation, and then at 3 monthly intervals until they were stable using daily lid cleaning alone.

Moderately severe or severe cases were treated with systemic erythromycin $30-40 \mathrm{mg} / \mathrm{kg}$ body weight (three divided doses for 3 weeks, then twice daily for 4-6 weeks), topical broad spectrum antibiotic eye drops (chloramphenicol four times daily for 4-6 weeks), and the application of

Abbreviations: BKC, blepharokeratoconjunctivitis 


\begin{tabular}{|lll|}
\hline Table 1 Classification of the severity of blepharokeratoconjunctivitis in children \\
\hline Mild & Lids/conjunctiva & $\begin{array}{l}\text { Corneal involvement } \\
\text { (degrees) }\end{array}$ \\
& $\begin{array}{l}\text { Minimal hyperaemia } \\
\text { Mild diffuse infiltration } \\
\text { Tarsal conjunctival vessels clearly visible } \\
\text { Mild papillary hyperplasia and small follicles (<5) }\end{array}$ & $<120$ \\
Moderate & $\begin{array}{l}\text { Moderate hyperaemia } \\
\text { Diffuse infiltration } \\
\text { Tarsal conjunctival vessels just visible } \\
\text { Moderate papillary hyperplasia and follicles (5-10) } \\
\text { Severe }\end{array}$ & $\begin{array}{l}\text { Marked hyperaemia } \\
\text { Marked diffuse infiltration } \\
\text { Tarsal conjunctival vessels not visible } \\
\text { Moderate papillary hyperplasia and follicles (>10) }\end{array}$ \\
\hline
\end{tabular}

chloramphenicol ointment to the lid margins at night to reduce the bacterial load. Topical corticosteroids (prednisolone $0.3-0.5 \%$, or fluorometholone $0.1 \%$, four times daily for 4-6 weeks, then decreasing the frequency to once or twice daily over $2-3$ months or longer) were used to control the conjunctival and corneal inflammation. When corneal phlyctens were present topical dexamethasone $0.1 \%$ was used two to four times daily for up to 2 months before decreasing the strength and frequency. These patients were examined at 2 and 4 weeks after presentation, then at 4-8 weekly intervals. Intraocular pressures were assessed in all cases.

\section{Statistical methods}

The distribution of clinical signs among the ethnic groups were evaluated by Pearson's $\chi^{2}$ test for association and the Fisher's exact test for small numbers, using the software package SPSS 11.5.1 for Windows.

\section{RESULTS}

During the 11 year study period, 44 children were identified with BKC. Patient demographics are shown in table 2. All children were born in the United Kingdom. In the final year of the study, 65 children attended the clinic, of whom 26 (40\%) had recurrent BKC, 23 (35.4\%) allergic conjunctivitis (perennial allergic conjunctivitis or vernal keratoconjunctvitis), and $16(24.6 \%)$ had a variety of other disorders. The median age at presentation was 5.4 (range 1-14) years, but the age of symptom onset antedated presentation by 5-18 months (median age 4.1 (range 1-14) years) (fig 2). This delay was attributable to previous treatment trials by referring general practitioners or local hospitals. The children were reviewed for a median of 7 years (range 7 months12 years), and the median age at discharge was 9.5 (6-15)

\begin{tabular}{|ll|}
\hline \multicolumn{2}{|l|}{ Table 2 Patient demographics } \\
\hline $\begin{array}{l}\text { Total number of patients } \\
\text { Sex (male: female) }\end{array}$ & 44 \\
Age (median (range) years) & $20: 24$ \\
at onset & $4.1(1-14)$ \\
at presentation & $5.4(1-14)$ \\
at discharge & $9.5(6-15)$ \\
Ethnic origin (n) & 20 \\
White & 22 \\
Asian* & 2 \\
Middlle Eastern & 0 \\
African-Caribbean & $7(0.58-12)$ \\
Follow up (median (range) (years)) & \\
\hline *Non-oriental children of Indian and Sri Lankan origin. There \\
were no children from Pakistan or Bangladesh. \\
\hline
\end{tabular}

years. BKC was more common in the Asian (Indian and Sri Lankan) population $(22(50 \%))$; this is a substantial overrepresentation of Asians as only 29\% of the London population comprised ethnic minorities in the 2001 census. All children had bilateral disease although there was marked asymmetry of symptoms and signs. The main symptoms are shown in table 3.

The most severe disease was seen in the Asian and Middle Eastern children $(\mathrm{p}<0.001)$ while the white group had significantly milder disease $(\mathrm{p}<0.001)$ (table 4$)$. The most common corneal features found in all ethnic groups were epithelial punctate keratitis and marginal infiltrates Although subepithelial punctate keratitis was also seen in $28(63.6 \%)$ patients, this was principally associated with the Asian and Middle Eastern groups $(p=0.008)$. Asian and Middle Eastern children were more likely to develop corneal vascularisation ( $p<0.001$ ), often involving all clock hours of the limbus $(p=0.008)$. These children were also more susceptible to marginal corneal ulceration $(p=0.003)$. Punctate erosions and corneal phlyctenules were restricted to the Asian subpopulation.

\begin{tabular}{|lc|}
\hline \multicolumn{2}{|c|}{ Table 3 Clinical symptoms and signs } \\
\hline & No (\%) \\
\hline Symptoms & \\
Redness & $21(47.7)$ \\
Watering & $22(50)$ \\
Itching & $10(22.7)$ \\
Grittiness & $24(54.5)$ \\
Discharge & $29(65.9)$ \\
Photophobia & $28(63.6)$ \\
Pain & $3(6.8)$ \\
Signs & \\
Lids/coniunctiva & \\
Blepharitis & $29(65.9)$ \\
Chalazia & $7(15.9)$ \\
Folliculitis & $6(13.6)$ \\
Meibomianitis & $3(6.8)$ \\
Styes & $3(6.8)$ \\
Hyperaemia & $21(47.7)$ \\
Bulbar conjunctival phlyctenules & $9(20.5)$ \\
Papillary hyperplasia & $22(50)$ \\
Follicular hyperplasia & $20(45.5)$ \\
Cornea & \\
Punctate erosions & $4(9.1)$ \\
Epithelial punctate keratitis & $26(59.1)$ \\
Subepithelial punctate keratitis & $28(63.6)$ \\
Marginal Infiltrates & $28(63.6)$ \\
Corneal phlyctenule & $2(4.6)$ \\
Sectoral vascularisation & $15(34.1)$ \\
Circumcorneal vascularisation & $12(27.3)$ \\
\hline & \\
\hline & \\
\hline
\end{tabular}



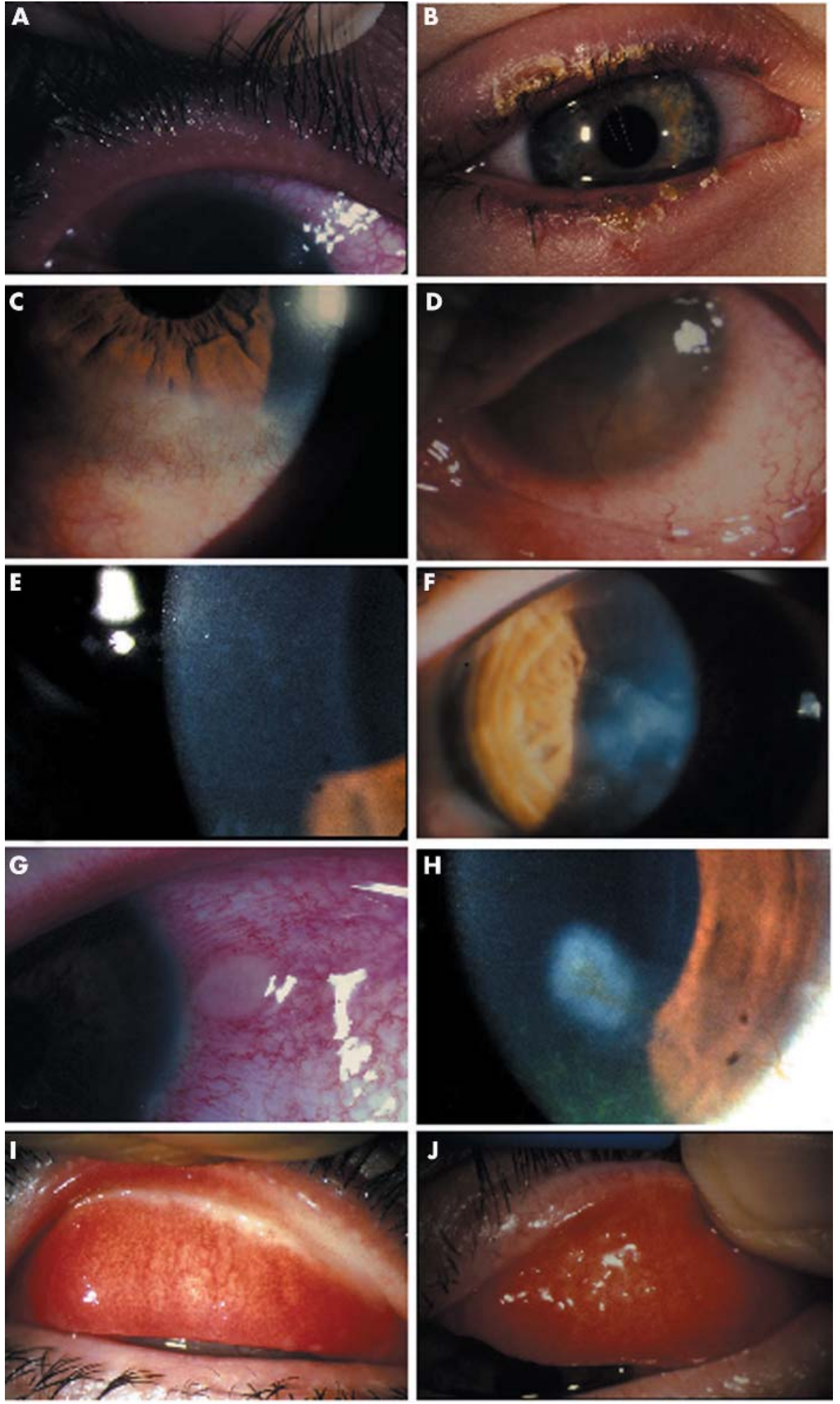

$\mathbf{H}$
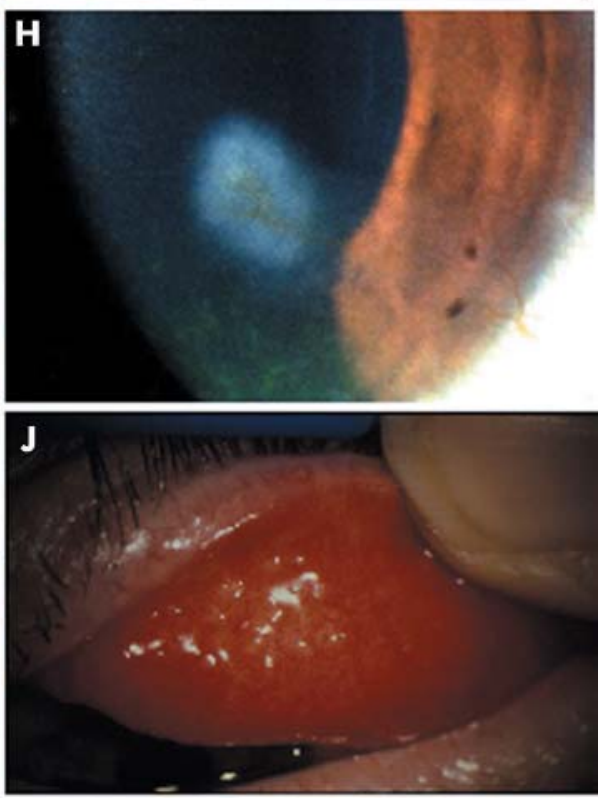

Figure 1 Clinical signs seen in staphylococcal blepharokeratoconjuctivitis. (A) Blepharitis;

(B) ulcerative blepharitis with marked collarettes; (C) sectoral marginal vascularisation and keratitis; (D) severe corneal vascularisation and keratitis; (E) subepithelial keratitis; (F) central corneal scar; $(G)$ conjunctival phlyctenule; $(H)$ resolved corneal phlyctenule with residual scarring and corneal vascularisation (I) upper tarsus (mild to moderate inflammation); and (J) upper tarsus (severe inflammation).
Fifteen $(34.1 \%)$ children had culture positive lid margin and conjunctival swabs. Of these, 12 had a moderate or heavy growth of Staphylococcus aureus, one $S$ epidermidis, and two mixed $S$ aureus/S epidermidis. All bacterial loads were sensitive to chloramphenicol and erythromycin. Clinically, these children had moderate or severe disease and were predominantly Asian.

With treatment, the children had a reduction in symptoms and signs, and continuation of the disease after the age of 8 was uncommon (fig 2). In the most severe cases, long term, low dose topical corticosteroid therapy was used to prevent active inflammatory disease or corneal scarring.

\section{DISCUSSION}

Our data have shown that the clinical syndrome of BKC is an identifiable inflammatory external disease in children, in which the symptoms and signs are similar to those found in adults although the disease course differs. Children presented 


\begin{tabular}{|c|c|c|c|c|c|}
\hline & $\begin{array}{l}\text { All patients } \\
\text { No (\%) }\end{array}$ & $\begin{array}{l}\text { White } \\
\text { No (\%) }\end{array}$ & $\begin{array}{l}\text { Asian } \\
\text { No (\%) }\end{array}$ & $\begin{array}{l}\text { Middle East } \\
\text { No }(\%)\end{array}$ & p Value \\
\hline $\begin{array}{l}\text { Number of patients } \\
\text { Severity of disease }\end{array}$ & 44 & 20 & 22 & 2 & - \\
\hline Mild & $21(47.7)$ & $18(90.0)$ & $3(13.6)$ & $0(0)$ & $<0.001$ \\
\hline Moderate & $11(25.0)$ & $2(10.0)$ & $9(40.9)$ & $0(0)$ & 0.03 \\
\hline Severe & $12(27.2)$ & $0(0)$ & $10(45.5)$ & $2(100))$ & $<0.001$ \\
\hline Punctate erosions & $4(9.1)$ & $0(0)$ & $4(18.2)$ & $0(0)$ & NS \\
\hline Epithelial punctate keratitis & $26(59.1)$ & 11 (55) & $13(59.1)$ & $2(100)$ & NS \\
\hline Subepithelial punctate keratitis & $28(63.6)$ & $8(40)$ & $18(81.8)$ & $2(100)$ & 0.008 \\
\hline Vascularisation & $27(61.4)$ & $6(30)$ & $19(86.4)$ & $2(100)$ & $<0.001$ \\
\hline Sectoral & $15(34.1)$ & $6(30)$ & $9(40.9)$ & $0(0)$ & NS \\
\hline Circumcorneal & $12(27.2)$ & $0(0)$ & $10(45.5)$ & $2(100))$ & $<0.001$ \\
\hline Marginal infiltrates & $28(63.6)$ & $12(60)$ & $14(63.6)$ & $2(100)$ & NS \\
\hline Marginal ulcers & $4(9.1)$ & $0(0)$ & $2(9.1)$ & $2(100)$ & 0.003 \\
\hline Corneal plyctenules & $2(4.6)$ & $0(0)$ & $2(9.1)$ & $0(0)$ & NS \\
\hline
\end{tabular}

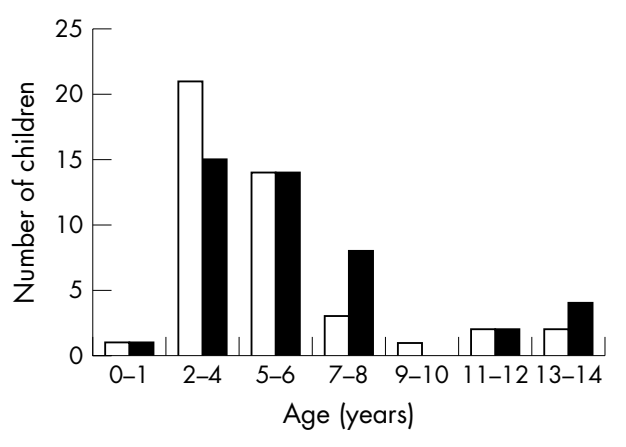

Figure 2 Distribution of the age at onset of symptoms and presentation. Open bars, age at onset of symptoms; solid bars, age at presentation; $n=44$.

between the ages of 1 and 14 (median 5.4) years with chronic redness and irritation that resulted in constant eye rubbing and photophobia. During our follow up period of 7 (range 0.58-12) years, the symptoms and signs reduced with treatment, and progression after the age of 8 was rare. The most severe cases, however, required long term therapy. These findings were similar to those of Farpour et al who followed eight children with BKC for a shorter period of 8.3 (range 2-23) months. By contrast, their cohort was younger ( 3.2 years (range $0.5-8$ years )), and relief of symptoms and signs occurred within 2 months of treatment; however, all had recurrences within the follow up period requiring repeat therapy and long term treatment was not used.

Asian and Middle Eastern children were most severely affected and often had $S$ aureus growth on lid or conjunctival cultures. There is no clear explanation for this finding. Our treatment strategies were aimed at (i) reducing the stimulus for the disease by lowering the local population of staphylococci, (ii) improving meibomian gland dysfunction, and (iii) reducing the host's local immune response. This was achieved by the therapy described in the methods section above. The preferred systemic antibiotic was erythromycin. This is safe for use in children under the age of 8 years,,$^{5-7}$ and has previously been used in small case series of children with BKC. ${ }^{5}{ }^{6}$ Like the tetracyclines in adults, ${ }^{8}{ }^{9}$ macrolides, such as erythromycin, appear to penetrate and accumulate in the meibomian glands affecting sebum composition, as well as inhibiting bacterial protein synthesis and lipase production. It is unclear if these antibiotics also alter the ocular flora or an antigenically stimulated immune response.

In summary, our data have characterised the syndrome of childhood BKC, and have found it to be a common disease in the children in our corneal and external diseases clinics at our tertiary referral centre. The most severe disease occurred in the Asian and Middle Eastern populations. Progression was rare after the age of 8 although long term treatment was needed until age 15 in a few children. Further studies to define the immunopathogenesis and genetic components of this disease would be appropriate.

\section{Authors' affiliations}

M Viswalingam, S Rauz, N Morlet, J K G Dart, Corneal and External Diseases Service, Moorfields Eye Hospital NHS Trust, City Road, London ECIV 2PD, UK

The authors have no proprietary interest in the products described in this article.

Correspondence to: John K G Dart, Corneal and External Diseases Service, Moorfields Eye Hospital NHS Trust, 162 City Road, London ECIV 2PD, UK; i.dart@ucl.ac.uk

Accepted for publication 29 August 2004

\section{REFERENCES}

1 Thygeson P. Bacterial factors in chronic catarrhal conjunctivitis: the role of toxin forming staphylococci. Arch Ophthalmol 1937;18:373-87.

2 McCulley JP, Dougherty JM, Deneau DG. Classification of chronic blepharitis. Ophthlamology 1987;89:1173-80.

3 McCulley JP. Blepharoconjunctivitis: common corneal problems. In: Olson RJ, ed. International Ophthalmology Clinics 1989;24(2).

4 Wilhelmus KR. Inflammatory disorders of the eyelid margins and lashes. Ophthalmol Clin North Am 1992;5:187.

5 Farpour B, McClellan KA. Diagnosis and management of chronic blepharoconjunctivitis in children. J Paediatr Ophthalmol Strabismus 2001;38:204-12

6 Meisler DM, Raizman MB, Traboulsi El. Oral erythromycin treatment for childhood blepharokeratitis. J AAPOS 2000;4:379-80.

7 Klein JO. History of macrolide use in paediatrics Paediatr Infect Dis J 1997; 16:427-31.

8 Zaidman GW, Brown SI. Orally administered tetracycline for phlyctenular kerato-conjunctivitis. Am J Ophthalmol 1981;92:173-82.

9 Doherty JM, McCulley JP, Silvany RE, et al. The role of tetracycline in chronic blepharitis: inhibition of lipase production in staphylococci. Invest Ophthal Vis Sci 1991;32:2970-5. 